\title{
Parameter estimation for stochastic equations with additive fractional Brownian sheet
}

\author{
Tommi Sottinen* Ciprian A. Tudor ${ }^{\dagger}$
}

June 21, 2007

\begin{abstract}
We study the maximum likelihood estimator for stochastic equations with additive fractional Brownian sheet. We use the Girsanov transform for the the two-parameter fractional Brownian motion, as well as the Malliavin calculus and Gaussian regularity theory.
\end{abstract}

Mathematics Subject Classification (2000): 60G15, G0H07, 60G35, 62M40

Key words and phrases: maximum likelihood estimator, fractional Brownian sheet, Malliavin calculus, Girsanov transform

\section{Introduction}

The recent development in the stochastic calculus with respect to the fractional Brownian motion has led to the study of parameter estimation problems for stochastic equations driven by this process. Several authors has studied these aspects; see e.g. $[8,9,19,10,25]$. An obvious extension is to study the two-parameter case. Elements of the stochastic calculus with respect to the fractional Brownian sheet has recently been considered by $[23,24]$ and stochastic equations with fractional Brownian sheet has emerged in [3] and [21].

The aim of this work is to construct and to study the asymptotic behavior of the maximum likelihood estimator (MLE) for the parameter $\theta$ in the equation

$$
X_{t, s}=\theta \int_{0}^{t} \int_{0}^{s} b\left(X_{v, u}\right) \mathrm{d} u \mathrm{~d} v+W_{t, s}^{\alpha, \beta}, \quad t, s \in[0, T]
$$

where $W^{\alpha, \beta}$ is a fractional Brownian sheet with Hurst parameters $\alpha, \beta \in(0,1)$ and $b$ is a Lipschitz function. Our construction of the estimator is based on the Girsanov transform and uses the connection between the fractional Brownian sheet and the standard one, Malliavin calculus, and Gaussian regularity theory. A related work on a two-parameter model with standard Brownian sheet is the paper [2].

\footnotetext{
${ }^{*}$ Reykjavik University, Kringlan 1, 103 Iceland, tommi@ru.is

${ }^{\dagger}$ SAMOS-MATISSE, Université de Panthéon-Sorbonne Paris 1, 90, rue de Tolbiac, 75634 Paris Cedex 13, France, tudor@univ-paris1.fr
} 
Besides its theoretical interest situation in the context of the stochastic calculus for Gaussian processes and fields, our work is also motivated by practical aspects. Models of the form (1.1) traditionally appears in signal estimation problem: the coefficient of the signal $\theta$ is unobservable and it has to be estimated from the observation of the process $X$ which represents the signal plus the noise. Such two-dimensional models where the noise has fractional behavior has been recently considered in satellite imaging (see [18]): the transmitted image is disturbed by external factors whose properties are related to those of fractional Brownian sheet. Other areas where such models driven by fractional Brownian sheets appear are: radar image data classification (see [7]), the classification and segmentation of hydrological basins (see [13]) medical applications like early detection of osteoroposis from X-ray images (see [12], $[6])$.

The paper is organized as follows. Section 2 contains some preliminaries on the fractional Brownian sheet. In Section 3, using the techniques of the Malliavin calculus, we prove that the solution is sub-Gaussian. Section 4 contains the proof of the existence of the MLE for the parameter $\theta$ and we separate this proof following the values of $\alpha$ and $\beta$. Finally, in Section 5, we present a different expression of the MLE that relates our work with the approach of [9]. This form of the estimator will be used to obtain the the strong consistency of the MLE in the linear case $b(x)=x$.

\section{Fractional Brownian sheet as a Volterra sheet}

Let $T>0$. The Brownian sheet is a two-parameter Gaussian process $\left(W_{t, s}\right)_{t, s \in[0, T]}$, starting from zero, with mean zero and covariance function

$$
\mathbf{E}\left(W_{t, s} W_{v, u}\right)=(t \wedge v)(s \wedge u), \quad t, s, v, u \in[0, T] .
$$

The (anisotropic) fractional Brownian sheet with Hurst index $(\alpha, \beta) \in(0,1)^{2}$ is a two-parameter Gaussian process $\left(W_{t, s}^{\alpha, \beta}\right)_{t, s \in[0, T]}$, starting from zero, with mean zero and covariance function

$$
\mathbf{E}\left(W_{t, s}^{\alpha, \beta} W_{v, u}^{\alpha, \beta}\right)=\frac{1}{2}\left(t^{2 \alpha}+v^{2 \alpha}-|t-v|^{2 \alpha}\right) \frac{1}{2}\left(s^{2 \beta}+u^{2 \beta}-|s-u|^{2 \beta}\right),
$$

$t, s, v, u \in[0, T]$.

We recall how the fractional Brownian sheet $W^{\alpha, \beta}$ can be represented by a standard Brownian sheet $W=W^{\frac{1}{2}, \frac{1}{2}}$ that is constructed from it. For details and references see $[20,21]$.

We start with the one-parameter case. Define a Volterra kernel (i.e. a kernel which vanishes if the second variable is greater than the first one)

$$
K_{\alpha}(t, s)=c_{\alpha}\left(\left(\frac{t}{s}\right)^{\alpha-\frac{1}{2}}(t-s)^{\alpha-\frac{1}{2}}-\left(\alpha-\frac{1}{2}\right) s^{\frac{1}{2}-\alpha} \int_{s}^{t} u^{\alpha-\frac{3}{2}}(u-s)^{\alpha-\frac{1}{2}} \mathrm{~d} u\right),
$$

where the normalising constant is

$$
c_{\alpha}=\sqrt{\frac{\left(2 \alpha+\frac{1}{2}\right) \Gamma\left(\frac{1}{2}-\alpha\right)}{\Gamma\left(\alpha+\frac{1}{2}\right) \Gamma(2-2 \alpha)}}
$$


and $\Gamma$ is the Euler's gamma function. It was shown in [14] that the fractional Brownian motion $W^{\alpha}$ with Hurst index $\alpha \in(0,1)$ can be represented by using a standard Brownian motion $W=W^{\frac{1}{2}}$ as

$$
W_{t}^{\alpha}=\int_{0}^{t} K_{\alpha}(t, s) \mathrm{d} W_{s}
$$

This Wiener integral can be understood both in pathwise and $L^{2}$-sense. The Brownian motion $W$ is actually constructed from the fractional one by the (pathwise or $L^{2}$ ) integral

$$
W_{t}=\int_{0}^{t} K_{\alpha}^{-1}(t, s) \mathrm{d} W_{s}^{\alpha} .
$$

Here the Volterra kernel $K_{\alpha}^{-1}$ is

$$
\begin{gathered}
K_{\alpha}^{-1}(t, s)=c_{\alpha}^{\prime}\left(\left(\frac{t}{s}\right)^{\alpha-\frac{1}{2}}(t-s)^{\frac{1}{2}-\alpha}-\left(\alpha-\frac{1}{2}\right) s^{\frac{1}{2}-\alpha} \int_{s}^{t} u^{\alpha-\frac{3}{2}}(u-s)^{\frac{1}{2}-\alpha} \mathrm{d} u\right), \\
c_{\alpha}^{\prime}=\frac{\Gamma\left(\alpha+\frac{1}{2}\right) \Gamma(2-2 \alpha)}{\mathrm{B}\left(\frac{1}{2}-\alpha\right) \sqrt{\left(2 \alpha+\frac{1}{2}\right) \Gamma\left(\frac{1}{2}-\alpha\right)}},
\end{gathered}
$$

and $\mathrm{B}$ is the beta function.

Now we turn to the two-parameter case. Let

$$
\begin{aligned}
& K_{\alpha, \beta}(t, s ; v, u)=K_{\alpha}(t, v) K_{\beta}(s, u), \\
& K_{\alpha, \beta}^{-1}(t, s ; v, u)=K_{\alpha}^{-1}(t, v) K_{\beta}^{-1}(s, u) .
\end{aligned}
$$

The kernels $K_{\alpha, \beta}$ and $K_{\alpha, \beta}^{-1}$ are of Volterra type: they vanish if $v \geq t$ or $u \geq s$. Then from the one-parameter case it follows that we have the following (pathwise and $L^{2}$-sense) transformations connecting the fractional Brownian sheet $W^{\alpha, \beta}$ and the standard one $W=W^{\frac{1}{2}, \frac{1}{2}}$ :

$$
\begin{aligned}
W_{t, s}^{\alpha, \beta} & =\int_{0}^{t} \int_{0}^{s} K_{\alpha, \beta}(t, s ; v, u) \mathrm{d} W_{u, v} \\
W_{t, s} & =\int_{0}^{t} \int_{0}^{s} K_{\alpha, \beta}^{-1}(t, s ; v, u) \mathrm{d} W_{u, v}^{\alpha, \beta} .
\end{aligned}
$$

We shall use this connection in the following way: The fractional Brownian sheet $W^{\alpha, \beta}$ is assumed to be given, the standard Brownian sheet $W$ is constructed from the fractional one $W^{\alpha, \beta}$ by the formula (2.2), and then $W^{\alpha, \beta}$ is represented in terms of $W$ by the formula (2.1).

In what follows we shall denote by $K_{\alpha, \beta}$ also the operator on $L^{2}\left([0, T]^{2}\right)$ induced by the kernel $K_{\alpha, \beta}$ :

$$
K_{\alpha, \beta}[f](t, s)=\int_{0}^{t} \int_{0}^{s} K_{\alpha, \beta}(t, s ; v, u) f(v, u) \mathrm{d} u \mathrm{~d} v,
$$


and similarly for $K_{\alpha, \beta}^{-1}$. Note that as an operator $K_{\alpha, \beta}^{-1}$ is indeed the inverse of the operator $K_{\alpha, \beta}$.

Finally, we note the following connection with deterministic fractional calculus. The fractional Riemann-Liouville integral of order $\gamma>0$ is

$$
I^{\gamma}[f](t)=\frac{1}{\Gamma(\gamma)} \int_{0}^{t}(t-v)^{\gamma-1} f(v) \mathrm{d} v
$$

The fractional Weyl derivative of order $\gamma \in(0,1)$ is

$$
I^{-\gamma}[f](t)=\frac{1}{\Gamma(1-\gamma)}\left[\frac{f(t)}{t^{\gamma}}+\gamma \int_{0}^{t} \frac{f(t)-f(v)}{(t-v)^{\gamma+1}} \mathrm{~d} v\right] .
$$

The mixed fractional integral-differential operator $I^{\gamma, \eta}$ acts on two-argument functions $f=f(t, s)$ argumentwise: $I^{\gamma, \eta}[f](t, s)=I^{\gamma}\left[f_{\eta}(\cdot, s)\right](t)$, where $f_{\eta}(t, s)=$ $I^{\eta}[f(t, \cdot)](s)$. Now, we have the representation (see $[3]$ )

$$
K_{\alpha, \beta}^{-1}[f](t, s)=c_{\alpha, \beta}^{\prime \prime} t^{\alpha-\frac{1}{2}} s^{\beta-\frac{1}{2}} I^{\frac{1}{2}-\alpha, \frac{1}{2}-\beta}\left[t^{\frac{1}{2}-\alpha} s^{\frac{1}{2}-\beta} \frac{\partial^{2} f}{\partial t \partial s}\right](t, s),
$$

where $c_{\alpha, \beta}^{\prime \prime}$ is a certain normalising constant.

\section{On the solution}

Now we shall focus our attention to the stochastic differential equation

$$
X_{t, s}=\theta \int_{0}^{t} \int_{0}^{s} b\left(X_{v, u}\right) \mathrm{d} u \mathrm{~d} v+W_{t, s}^{\alpha, \beta}, \quad t, s \in[0, T] .
$$

The equation (3.1) has been considered in [3] for parameters $(\alpha, \beta) \in\left(0, \frac{1}{2}\right)^{2}$ in the more general context $b(x)=b(t, s ; x)$ with $t, s \in[0, T]$. It has been proved that, if $b$ satisfy the linear growth condition

$$
|b(t, s ; x)| \leq C(1+|x|)
$$

then (3.1) admits a unique weak solution, and if $b$ is nondecreasing in the second variable and bounded, then (3.1) has a unique strong solution.

We are here interesting in the case when the drift coefficient $b$ is Lipschitz (more exactly, we will assume that $b$ is differentiable with bounded derivative). It is clear that in this case the method of standard Picard iterations can be applied to obtain the existence and the uniqueness of the solution for all Hurst parameters $\alpha, \beta$ belonging to $(0,1)$. As far as we know there are not existence and uniqueness results in the non-Lipschitz case if $\alpha$ or $\beta$ are bigger than $\frac{1}{2}$.

Since our main objective is the construction of a maximum likehood estimator from the observation of the trajectory of the process $X$ that satisfies (3.1), we will need some estimates on the supremum of this processes, and even more generally, on the variations of this process. For this purpose, we follow the ideas developped in [25] to prove that the solution of (3.1) is a sub-Gaussian process with respect to 
a certain canonical metric. The proof is based on the Poincaré inequality and uses some elements of the Malliavin calculus.

We make the following assumption on the drift $b$ throughout the rest of the paper:

(C1) $b$ is differentiable with bounded derivative.

Denote by $D$ the Malliavin derivative with respect to the Brownian sheet $W$. We refer to [16] details of Malliavin calculus and just recall two basic facts:

(i) If $F$ is a Wiener integral of the form

$$
F=\int_{0}^{T} \int_{0}^{T} f(t, s) \mathrm{d} W_{s, t}
$$

with $f \in L^{2}\left([0, T]^{2}\right)$ then

$$
D_{t, s} F=f(t, s) \text {. }
$$

(ii) If $F$ is a random variable differentiable in the Malliavin sense and $b$ is a function satisfying the condition $(\mathrm{C} 1)$ then $b(F)$ is Malliavin differentiable and we have the chain rule

$$
D_{t, s} b(F)=b^{\prime}(F) D_{t, s} F .
$$

We will need two auxiliary lemmas.

3.2 Lemma. There exists a constant $C_{\theta}$ depending on $T, \alpha, \beta,\left\|b^{\prime}\right\|_{\infty}$ and the parameter $\theta$ such that for every $t, s \in[0, T]$ we have the bound

$$
\left\|D ., X_{t, s}\right\|_{L^{2}\left(\Omega \times[0, T]^{2}\right)}^{2} \leq C_{\theta} .
$$

Proof. Taking Malliavin derivatives $D_{v, u}$ on the both sides of the equation (3.1) we obtain

$$
D_{v, u} X_{t, s}=\theta \int_{v}^{t} \int_{u}^{s} b^{\prime}\left(X_{v^{\prime}, u^{\prime}}\right) D_{v, u} X_{v^{\prime}, u^{\prime}} \mathrm{d} u^{\prime} \mathrm{d} v^{\prime}+K_{\alpha, \beta}(t, s ; v, u) .
$$

Denote

$$
M_{t, s}=\int_{0}^{T} \int_{0}^{T}\left|D_{v, u} X_{t, s}\right|^{2} \mathrm{~d} u \mathrm{~d} v=\int_{0}^{t} \int_{0}^{s}\left|D_{v, u} X_{t, s}\right|^{2} \mathrm{~d} u \mathrm{~d} v
$$


Then, by Fubini theorem and the estimate $(x+y)^{2} \leq 2 x^{2}+2 y^{2}$, we obtain

$$
\begin{aligned}
M_{t, s}= & \theta^{2} \int_{0}^{t} \int_{0}^{s}\left|\int_{v}^{t} \int_{u}^{s} b^{\prime}\left(X_{v^{\prime}, u^{\prime}}\right) D_{v, u} X_{v^{\prime}, u^{\prime}} \mathrm{d} u^{\prime} \mathrm{d} v^{\prime}+K_{\alpha, \beta}(t, s ; v, u)\right|^{2} \mathrm{~d} u \mathrm{~d} v \\
\leq & 2 \theta^{2} \int_{0}^{t} \int_{0}^{s}\left|\int_{v}^{t} \int_{u}^{s} b^{\prime}\left(X_{v^{\prime}, u^{\prime}}\right) D_{v, u} X_{v^{\prime}, u^{\prime}} \mathrm{d} u^{\prime} \mathrm{d} v^{\prime}\right|^{2} \mathrm{~d} u \mathrm{~d} v \\
& +2 \int_{0}^{t} \int_{0}^{s} K_{\alpha, \beta}(t, s ; v, u)^{2} \mathrm{~d} u \mathrm{~d} v \\
\leq & 2 \theta^{2}\left\|b^{\prime}\right\|_{\infty}^{2} \int_{0}^{t} \int_{0}^{s}\left\{\int_{v}^{t} \int_{u}^{s}\left|D_{v, u} X_{v^{\prime}, u^{\prime}}\right|^{2} \mathrm{~d} u^{\prime} \mathrm{d} v^{\prime}\right\} \mathrm{d} u \mathrm{~d} v+2 t^{2 \alpha} s^{2 \beta} \\
= & 2 \theta^{2}\left\|b^{\prime}\right\|_{\infty}^{2} \int_{0}^{t} \int_{0}^{s}\left\{\int_{0}^{v^{\prime}} \int_{0}^{u^{\prime}}\left|D_{v, u} X_{v^{\prime}, u^{\prime}}\right|^{2} \mathrm{~d} u \mathrm{~d} v\right\} \mathrm{d} u^{\prime} \mathrm{d} v^{\prime}+2 t^{2 \alpha} s^{2 \beta} \\
= & 2 \theta^{2}\left\|b^{\prime}\right\|_{\infty}^{2} \int_{0}^{t} \int_{0}^{s} M_{v^{\prime}, u^{\prime}} \mathrm{d} u^{\prime} \mathrm{d} v^{\prime}+2 t^{2 \alpha} s^{2 \beta} .
\end{aligned}
$$

So, the claim follows by a two-parameter version of the Gronwall lemma.

3.4 Lemma. Let $X$ be the unique solution of (3.1). Then there exists a constant $C_{\theta}$ depending on $T, \alpha, \beta,\left\|b^{\prime}\right\|_{\infty}$ and the parameter $\theta$ such that for every $s \leq s^{\prime}, t \leq t^{\prime}$ it holds that

$$
\left\|D_{., .}\left(X_{t^{\prime}, s^{\prime}}-X_{t, s}\right)\right\|_{L^{2}\left(\Omega \times[0, T]^{2}\right)}^{2} \leq C_{\theta}\left(\left|t-t^{\prime}\right|^{2 \alpha}+\left|s-s^{\prime}\right|^{2 \beta}\right) .
$$

Proof. For every $s \leq s^{\prime}$ and $t \leq t^{\prime}$, we have

$$
X_{t^{\prime}, s^{\prime}}-X_{t, s}=X_{t^{\prime}, s^{\prime}}-X_{t, s^{\prime}}+X_{t, s^{\prime}}-X_{t, s}
$$

So, it is enough to show that

$$
\left\|D ., \cdot\left(X_{t^{\prime}, s^{\prime}}-X_{t, s^{\prime}}\right)\right\|_{L^{2}\left(\Omega \times[0, T]^{2}\right)}^{2} \leq C_{\theta}\left(\left|t-t^{\prime}\right|^{2 \alpha}\right) .
$$

Now

$$
X_{t^{\prime}, s^{\prime}}-X_{t, s^{\prime}}=\theta \int_{t}^{t^{\prime}} \int_{0}^{s^{\prime}} b\left(X_{v, u}\right) \mathrm{d} u \mathrm{~d} v+W_{t^{\prime}, s^{\prime}}^{\alpha, \beta}-W_{t, s^{\prime}}^{\alpha, \beta}
$$

and thus

$$
\begin{aligned}
D_{a, b}\left[X_{t^{\prime}, s^{\prime}}-X_{t, s^{\prime}}\right]= & \theta \int_{t}^{t^{\prime}} \int_{0}^{s^{\prime}} b^{\prime}\left(X_{v, u}\right) D_{a, b} X_{v, u} \mathrm{~d} u \mathrm{~d} v \\
& +K_{\beta}\left(s^{\prime}, b\right)\left(K_{\alpha}\left(t^{\prime}, a\right)-K_{\alpha}(t, a)\right) .
\end{aligned}
$$

By using the fact that

$$
\int_{0}^{T}\left(K_{\alpha}\left(t^{\prime}, a\right)-K_{\alpha}(t, a)\right)^{2} \mathrm{~d} a=\left|t^{\prime}-t\right|^{2 \alpha}
$$


we obtain

$$
\begin{aligned}
& \mathbf{E}\left[\int_{0}^{T} \int_{0}^{T}\left|D_{a, b}\left[X_{t^{\prime}, s^{\prime}}-X_{t, s^{\prime}}\right]\right|^{2} \mathrm{~d} b \mathrm{~d} a\right] \\
& \leq 2 \theta^{2} \mathbf{E}\left[\left|\int_{t}^{t^{\prime}} \int_{0}^{s^{\prime}} b^{\prime}\left(X_{v, u}\right) D_{a, b} X_{v, u} \mathrm{~d} u \mathrm{~d} v\right|^{2} \mathrm{~d} b \mathrm{~d} a\right]+2\left(s^{\prime}\right)^{2 \beta}\left|t^{\prime}-t\right|^{2 \alpha} \\
& \leq 2 \theta^{2}\left\|b^{\prime}\right\|_{\infty}^{2} \mathbf{E}\left[\int_{0}^{T} \int_{0}^{T}\left|D_{a, b} X_{v, u}\right|^{2} \mathrm{~d} b \mathrm{~d} a\right]\left(t-t^{\prime}\right)^{2}+2\left(s^{\prime}\right)^{2 \beta}\left|t^{\prime}-t\right|^{2 \alpha} .
\end{aligned}
$$

The claim follows now from Lemma 3.2 and the fact that $\alpha, \beta<1$.

Recall that a sheet $X$ is sub-Gaussian with respect to metric $\delta$ if for all $\lambda \in \mathbb{R}$

$$
\mathbf{E}\left[\exp \left\{\lambda\left(X_{t, s}-X_{t^{\prime}, s^{\prime}}\right)\right\}\right] \leq \exp \left\{\frac{\lambda^{2}}{2} \delta\left(t, s ; t^{\prime}, s^{\prime}\right)^{2}\right\}
$$

3.7 Proposition. Suppose that $b$ satisfies condition (C1). Then the solution $X$ of (3.1) is a sub-Gaussian process with respect to the metric $\delta$ given by

$$
\delta\left(t, s ; t^{\prime}, s^{\prime}\right)^{2}=C_{\theta}\left(\left|t-t^{\prime}\right|^{2 \alpha}+\left|s-s^{\prime}\right|^{2 \beta}\right),
$$

where the constant $C_{\theta}$ comes from Lemma 3.4.

Proof. Recall the Poincaré inequality (see [26], page 76): if $F$ is a functional of the Brownian sheet $W$, then

$$
\mathbf{E}[\exp \{F\}] \leq \mathbf{E}\left[\exp \left\{\frac{\pi^{2}}{8}\|D F\|_{L^{2}\left([0, T]^{2}\right)}^{2}\right\}\right] .
$$

The claim follows from this and Lemma 3.4.

Proposition 3.7 says that, in the case of the Lipschitz coefficient $b$, the variations of the process $X$ are dominated, in distribution, by those of the Gaussian process with canonical metric (3.7); this prcosess is actually the so-called isotropic fractional Brownian sheet. As a consequence, the sub-Gaussian regularity theory (see [4] or [11]) can be applied to obtain supremum estimates on the process $X$. As an immediate consequence, we get, using the results in [11], Chapter 12 and the methods in [25]).

$$
\mathbf{E}\left[\sup _{v \leq t, u \leq s}\left|X_{v, u}\right|\right] \leq C_{\theta} \sqrt{t^{2 \alpha}+s^{2 \beta}} .
$$

and, for any positive $a_{\theta}=a\left(\alpha, \beta, T,\left\|b^{\prime}\right\|_{\infty}, \theta\right)$ small enough,

$$
\mathbf{E}\left[\exp \left\{a_{\theta} \sup _{t, s \in[0, T]}\left|X_{t, s}\right|^{2}\right\}\right]<\infty .
$$

These estimates will be explicitly used in the next section. 


\section{Maximum likelihood estimator}

First we recall how the Girsanov theorem for the shifted fractional Brownian sheet

$$
\tilde{W}_{t, s}^{\alpha, \beta}=W_{t, s}^{\alpha, \beta}+\int_{0}^{t} \int_{0}^{s} a_{u, v} \mathrm{~d} u \mathrm{~d} v
$$

can be recovered from the Girsanov theorem for the standard shifted Brownian sheet by the correspondence $(2.1)-(2.2)$.

Since the shift term in (4.1) is differentiable we can operate pathwise with the kernel $K_{\alpha, \beta}^{-1}$ on the both sides of the equation (4.1). So, we can set

$$
\tilde{W}_{t, s}=\int_{0}^{t} \int_{0}^{s} K_{\alpha, \beta}^{-1}(t, s ; v, u) \mathrm{d} \tilde{W}_{u, v}^{\alpha, \beta}
$$

and we have the following inverse relation for the transfer (4.2):

$$
\tilde{W}_{t, s}^{\alpha, \beta}=\int_{0}^{t} \int_{0}^{s} K_{\alpha, \beta}(t, s ; v, u) \mathrm{d} \tilde{W}_{u, v} .
$$

Now we want to find a shift $b$ such that

$$
\tilde{W}_{t, s}=W_{t, s}+\int_{0}^{t} \int_{0}^{s} b_{v, u} \mathrm{~d} u \mathrm{~d} v,
$$

where $W$ is a standard Brownian sheet constructed from the fractional one $W^{\alpha, \beta}$ by

$$
W_{t, s}=\int_{0}^{t} \int_{0}^{s} K_{\alpha, \beta}^{-1}(t, s ; v, u) \mathrm{d} W_{u, v}^{\alpha, \beta} .
$$

Comparing equations (4.1)-(4.5) we see that the connection is

$$
\int_{0}^{t} \int_{0}^{s} a_{v, u} \mathrm{~d} u \mathrm{~d} v=\int_{0}^{t} K_{\alpha, \beta}(t, s ; v, u) b_{v, u} \mathrm{~d} u \mathrm{~d} v
$$

So, we conclude that

$$
b_{t, s}=\int_{0}^{t} \int_{0}^{s} K_{\alpha, \beta}^{-1}(t, s ; v, u)\left(\int_{0}^{v} \int_{0}^{u} a_{v^{\prime}, u^{\prime}} \mathrm{d} u^{\prime} \mathrm{d} v^{\prime}\right) \mathrm{d} u \mathrm{~d} v
$$

or, in operator notation,

$$
b=K_{\alpha, \beta}^{-1}\left[\int_{0}^{\cdot} \int_{0}^{\cdot} a_{t, s} \mathrm{~d} s \mathrm{~d} t\right] .
$$

Now, comparing (4.1) to (4.4) with the connection (4.6) we obtain the following Girsanov theorem from the classical Girsanov theorem for the shifted standard Brownian sheet.

4.7 Theorem. Let $W^{\alpha, \beta}$ be a fractional Brownian sheet and let a be a process adapted to the filtration generated by $W^{\alpha, \beta}$. Let $W$ be a standard Brownian sheet constructed from $W^{\alpha, \beta}$ by (2.2) and let $b \quad b$ constructed from $a$ by (4.6). 
Assume that $b \in L^{2}\left(\Omega \times[0, T]^{2}\right)$, and $\mathbf{E}\left[V_{T, T}\right]=1$ where

$$
V_{T, T}=\exp \left\{-\int_{0}^{T} \int_{0}^{T} b_{t, s} \mathrm{~d} W_{s, t}-\frac{1}{2} \int_{0}^{T} \int_{0}^{T} b_{t, s}^{2} \mathrm{~d} s \mathrm{~d} t\right\} .
$$

Then under the new probability $\tilde{\mathbf{P}}$ with $\frac{\mathrm{d} \tilde{\mathbf{P}}}{\mathrm{d} \mathbf{P}}=V_{T, T}$ the process $\tilde{W}$ given by (4.2) is a Brownian sheet and the process $\tilde{W}^{\alpha, \beta}$ given by (4.1) is a fractional Brownian sheet.

The rest of this paper is devoted to construct a maximum likelihood estimator for the parameter $\theta$ in (3.1) by using the Girsanov theorem (Theorem 4.7). The existence and the expression of this MLE are given by the following result.

4.8 Proposition. Assume that one of the following holds:

(i) At least one of the parameters $\alpha$ and $\beta$ belongs to $\left(0, \frac{1}{2}\right)$ and $b$ satisfies (C1)

(ii) The parameters $\alpha$ and $\beta$ both belong to $\left(\frac{1}{2}, 1\right)$ and $b$ is linear.

Denote

$$
Q_{t, s}=K_{\alpha, \beta}^{-1}\left[\int_{0}^{\cdot} \int_{0}^{\cdot} b\left(X_{v, u}\right) \mathrm{d} u \mathrm{~d} v\right](t, s) .
$$

Then given observation over $[0, t]^{2}$ the $M L E$ for $\theta$ in (3.1) is

$$
\theta_{t}=-\frac{\int_{0}^{t} \int_{0}^{t} Q_{v, u} \mathrm{~d} W_{u, v}}{\int_{0}^{t} \int_{0}^{t} Q_{v, u}^{2} \mathrm{~d} u \mathrm{~d} v} .
$$

Before going into the proof of Proposition 4.8 let us note that we can also write

$$
\theta_{t}=\frac{\int_{0}^{t} \int_{0}^{t} Q_{v, u} \mathrm{~d} \tilde{W}_{u, v}}{\int_{0}^{t} \int_{0}^{t} Q_{v, u}^{2} \mathrm{~d} u \mathrm{~d} v} .
$$

This shows that the estimator can be deduced by the observed process $X$ since

$$
\tilde{W}_{t, s}=\int_{0}^{t} \int_{0}^{s} K_{\alpha, \beta}^{-1}(t, s ; v, u) \mathrm{d} X_{u, v} .
$$

Proof. Let us denote by $\mathbf{P}_{\theta}$ the law of the process $X_{t, s}$ that is the unique solution of (3.1). Then the MLE is obtained by taking the $\sup _{\theta} F_{\theta}$, where

$$
F_{\theta}=\frac{\mathrm{d} \mathbf{P}_{\theta}}{\mathrm{d} \mathbf{P}_{0}} .
$$

The conclusion (4.9) then follows by the Girsanov theorem (Theorem 4.7) if we show that $V_{t, t}$ is well-defined and $\mathbf{E}\left[V_{t, t}\right]=1$. The proof is separated into three cases. In what follows $\mathfrak{c}_{\alpha, \beta}$ is a generic constant depending on $\alpha$ and $\beta$ that may change even within a line. 
The case $\alpha, \beta \in\left(0, \frac{1}{2}\right)$ : In this case we use the expression (2.3) with $I^{\alpha-\frac{1}{2}, \beta-\frac{1}{2}}$ as a double fractional integral. We get

$$
Q_{t, s}=\mathfrak{c}_{\alpha, \beta} t^{\alpha-\frac{1}{2}} s^{\beta-\frac{1}{2}} \int_{0}^{t} \int_{0}^{s}(t-v)^{-\frac{1}{2}-\alpha} v^{\frac{1}{2}-\alpha}(s-u)^{-\frac{1}{2}-\beta} u^{\frac{1}{2}-\beta} b\left(X_{u, v}\right) \mathrm{d} u \mathrm{~d} v .
$$

Since $|b(x)| \leq|b(x)-b(0)|+|b(0)| \leq K\left(|x|+K^{\prime}\right)$, we see that

$$
\left|Q_{t, s}\right| \leq \mathfrak{c}_{\alpha, \beta}\left(K+\sup _{u \leq t, v \leq s}\left|X_{u, v}\right|\right) .
$$

Clearly (4.12) and the estimates (3.8), (3.9) show that $Q \in L^{2}\left([0, T]^{2}\right)$ and so $V_{t, t}$ is well-defined. To prove that $\mathbf{E}\left[V_{t, t}\right]=1$, it suffices to invoke Theorem 1.1, page 152 in [5] and to note that, by (4.12), there exists $a>0$ such that

$$
\sup _{v \leq t, u \leq s} \mathbf{E}\left[\exp \left\{a Q_{v, u}^{2}\right\}\right]<\infty
$$

The case $\alpha, \beta \in\left(\frac{1}{2}, 1\right)$ : In this case we use the operator $I^{\alpha-\frac{1}{2}, \beta-\frac{1}{2}}$ in the expression (2.3) is a double fractional derivative. We get

$$
\begin{aligned}
Q_{t, s}= & \mathfrak{c}_{\alpha, \beta} t^{\frac{1}{2}-\alpha} s^{\frac{1}{2}-\beta} b\left(X_{t, s}\right)+\mathfrak{c}_{\alpha, \beta} t^{\alpha-\frac{1}{2}} s^{\frac{1}{2}-\beta} \int_{0}^{t} \frac{t^{\frac{1}{2}-\alpha} b\left(X_{t, s}\right)-v^{\frac{1}{2}-\alpha} b\left(X_{v, s}\right)}{(t-v)^{\alpha+\frac{1}{2}}} \mathrm{~d} v \\
& +\mathfrak{c}_{\alpha, \beta} t^{\frac{1}{2}-\alpha} s^{\beta-\frac{1}{2}} \int_{0}^{s} \frac{s^{\frac{1}{2}-\beta} b\left(X_{t, s}\right)-u^{\frac{1}{2}-\beta} b\left(X_{t, u}\right)}{(s-u)^{\beta+\frac{1}{2}}} \mathrm{~d} u \\
& +\mathfrak{c}_{\alpha, \beta} \int_{0}^{t} \int_{0}^{s}\left\{t^{\frac{1}{2}-\alpha} v^{\frac{1}{2}-\beta} b\left(X_{t, s}\right)-v^{\frac{1}{2}-\alpha} v^{\frac{1}{2}-\beta} b\left(X_{v, s}\right)-t^{\frac{1}{2}-\alpha} u^{\frac{1}{2}-\beta} b\left(X_{t, u}\right)\right. \\
& \left.+v^{\frac{1}{2}-\alpha} u^{\frac{1}{2}-\beta} b\left(X_{v, u}\right)\right\}\left((t-v)^{-\alpha-\frac{1}{2}}(s-u)^{-\beta-\frac{1}{2}}\right) \mathrm{d} u \mathrm{~d} v \\
:= & A_{1}(t, s)+A_{2}(t, s)+A_{3}(t, s)+A_{4}(t, s) .
\end{aligned}
$$

The term $A_{1}(t, s)$ can be easily treated, since by $(\mathrm{C} 1)$,

$$
\left|A_{1}(t, s)\right| \leq \mathfrak{c}_{\alpha, \beta} t^{\frac{1}{2}-\alpha} s^{\frac{1}{2}-\beta}\left(K+\|X\|_{\infty}\right)
$$

which is finite by (4.12). The term $A_{2}(t, s)$ and $A_{3}(t, s)$ are rather similar to those appearing in the one-parameter case (see [17] and [25]). For the sake of completeness, we illustrate how to treat them. For $A_{2}(t, s)$ write

$$
\begin{aligned}
A_{2}(t, s)= & \mathfrak{c}_{\alpha, \beta} t^{\alpha-\frac{1}{2}} s^{\frac{1}{2}-\beta} b\left(X_{t, s}\right) \int_{0}^{t} \frac{t^{\frac{1}{2}-\alpha}-v^{\frac{1}{2}-\alpha}}{(t-v)^{\alpha+\frac{1}{2}}} \mathrm{~d} v \\
& +\mathfrak{c}_{\alpha, \beta} t^{\alpha-\frac{1}{2}} s^{\frac{1}{2}-\beta} \int_{0}^{t} \frac{b\left(X_{t, s}\right)-b\left(X_{v, s}\right)}{(t-v)^{\alpha+\frac{1}{2}}} \mathrm{~d} v \\
=: & A_{21}(t, s)+A_{22}(t, s) .
\end{aligned}
$$


Since

$$
\int_{0}^{t} \frac{t^{\frac{1}{2}-\alpha}-u^{\frac{1}{2}-\alpha}}{(t-v)^{\alpha+\frac{1}{2}}} \mathrm{~d} v=\mathfrak{c}_{\alpha, \beta} t^{1-2 \alpha},
$$

the summand $A_{21}(t, s)$ is clearly almost surely finite using condition $(\mathrm{C} 1)$. The second summand $A_{22}$ can be bounded as follows: for $\varepsilon$ small enough,

$$
\left|A_{2,2}(t, s)\right| \leq \mathfrak{c}_{\alpha, \beta} t^{\alpha-\frac{1}{2}} s^{\frac{1}{2}-\beta} \sup _{v \leq t}\left[\frac{\left|X_{t, s}-X_{v, s}\right|}{(t-v)^{\alpha-\varepsilon}}\right] \int_{0}^{t} u^{\frac{1}{2}-\alpha}(t-v)^{-\frac{1}{2}-\varepsilon} \mathrm{d} v
$$

By the Fernique theorem the supremum above has exponential moments. So, it follows that the Novikov criterium is satisfied. Let us study now the term $A_{4}(t, s)$. It is not difficult to see that the expression

$$
\begin{aligned}
I= & t^{\frac{1}{2}-\alpha} s^{\frac{1}{2}-\beta} b\left(X_{t, s}\right)-v^{\frac{1}{2}-\alpha} s^{\frac{1}{2}-\beta} b\left(X_{v, s}\right) \\
& -t^{\frac{1}{2}-\alpha} u^{\frac{1}{2}-\beta} b\left(X_{t, u}\right)+u^{\frac{1}{2}-\alpha} u^{\frac{1}{2}-\beta} b\left(X_{v, u}\right)
\end{aligned}
$$

can be written as

$$
\begin{aligned}
I= & \left(t^{\frac{1}{2}-\alpha}-u^{\frac{1}{2}-\alpha}\right) b\left(X_{t, s}\right)\left(s^{\frac{1}{2}-\beta}-v^{\frac{1}{2}-\beta}\right) \\
& +\left(t^{\frac{1}{2}-\alpha}-u^{\frac{1}{2}-\alpha}\right) v^{\frac{1}{2}-\beta}\left(b\left(X_{t, s}\right)-b\left(X_{t, v}\right)\right) \\
& +u^{\frac{1}{2}-\alpha}\left(s^{\frac{1}{2}-\beta}-v^{\frac{1}{2}-\beta}\right)\left(b\left(X_{t, s}\right)-b\left(X_{u, s}\right)\right) \\
& +u^{\frac{1}{2}-\alpha} v^{\frac{1}{2}-\beta}\left(b\left(X_{t, s}\right)-b\left(X_{t, v}\right)-b\left(X_{u, s}\right)+b\left(X_{u, v}\right)\right) .
\end{aligned}
$$

This gives a decomposition of the term $A_{4}(t, s)$ in four summands; The first three summands can be handled by using similar arguments to those already used throughout this proof. For the last term actually we need to assume the linearity of the function $b$ (and obviously the solution of (3.1) is then Gaussian). If $b$ is linear, then

$$
\begin{aligned}
& b\left(X_{t, s}\right)-b\left(X_{t, u}\right)-b\left(X_{v, s}\right)+b\left(X_{v, u}\right) \\
& =X_{t, s}-X_{t, u}-X_{v, s}+X_{v, u} \\
& =\int_{v}^{t} \int_{u}^{s} X_{b, a} \mathrm{~d} b \mathrm{~d} a+W^{\alpha, \beta}\left(\left(z, z^{\prime}\right]\right)
\end{aligned}
$$

where $W^{\alpha, \beta}\left(\left(z, z^{\prime}\right]\right)$ denotes the planar increments of $W^{\alpha, \beta}$ between $z=(v, u)$ and $z^{\prime}=(t, s)$. We finish again by an application of the Fernique theorem and observing that the process $W^{\alpha, \beta}$ (and thus $X$ ) is Hölder continuous of order $(\alpha, \beta)$ (see Proposition 5 in [1]).

The case $\alpha \in\left(0, \frac{1}{2}\right), \beta \in\left(\frac{1}{2}, 1\right)$ : In this case, it is not difficult to see that

$$
\begin{aligned}
Q_{t, s}= & \mathfrak{c}_{\alpha, \beta} t^{\alpha-\frac{1}{2}} \int_{0}^{t}(t-v)^{-\alpha-\frac{1}{2}} v^{\frac{1}{2}-\alpha} \\
& \times\left[s^{\frac{1}{2}-\beta} b\left(X_{v, s}\right)+\mathfrak{c}_{\alpha, \beta} s^{\beta-\frac{1}{2}} \int_{0}^{s} \frac{b\left(X_{v, s}\right) s^{\frac{1}{2}-\beta}-b\left(X_{v, u}\right) u^{\frac{1}{2}-\beta}}{(s-u)^{\frac{1}{2}+\beta}} \mathrm{d} u\right] \mathrm{d} v .
\end{aligned}
$$


Clearly, this case can be handled by combining the methods used in the first two cases. The only thing we need to note here is that the Fernique theorem holds for the sub-Gaussian process $X$ (actually, here we can use the fact that for each $v$, the process $s \mapsto X_{v, s}$ is sub-Gaussian with respect the metric $\left|s^{\prime}-s\right|^{\beta}$, see the proof of Lemma 2).

\section{Alternative form of the estimator}

We will try to relate here our approach with the one considered by [9] in the oneparameter scale.

As in the one -parameter case (see [14]) one can associate to the fractional Brownian sheet a two-parameter martingale (the so-called fundamental martingale). We refer to [22] for the two-parameter case. More precisely, let us define the deterministic function

$$
k_{\alpha}(t, u)=c_{\alpha}^{-1} u^{\frac{1}{2}-\alpha}(t-u)^{\frac{1}{2}-\alpha}, \quad c_{\alpha}=2 \alpha \Gamma\left(\frac{3}{2}-\alpha\right) \Gamma\left(\alpha+\frac{1}{2}\right)
$$

and

$$
\omega_{t}^{\alpha}=\lambda_{\alpha}^{-1} t^{2-2 \alpha}, \quad \lambda_{\alpha}=\frac{2 \Gamma(3-2 \alpha) \Gamma\left(\alpha+\frac{1}{2}\right)}{\Gamma\left(\frac{3}{2}-\alpha\right)} .
$$

Then the process

$$
M_{t, s}^{\alpha, \beta}=\int_{0}^{t} \int_{0}^{s} k_{\alpha}(t, v) k_{\beta}(s, u) \mathrm{d} W_{u, v}^{\alpha, \beta}
$$

is a two-parameter Gaussian (strong) martingale with quadratic variation equal to $\omega_{t}^{\alpha} \omega_{s}^{\beta}$ (the stochastic integral in (5.1) can be defined in a Wiener sense with respect to the fractional Brownian sheet). The filtration generated by $M^{\alpha, \beta}$ coincides to the one generated by $W^{\alpha, \beta}$.

Let us integrate the deterministic kernel $k_{\alpha}(t, v) k_{\beta}(s, u)$ with respect to both sides of (3.1). We get

$$
\begin{aligned}
Z_{t, s} & :=\int_{0}^{t} \int_{0}^{s} k_{\alpha}(t, v) k_{\beta}(s, u) \mathrm{d} X_{v, u} \\
& =\int_{0}^{t} \int_{0}^{s} k_{\alpha}(t, v) k_{\beta}(s, u) b\left(X_{v, u}\right) \mathrm{d} u \mathrm{~d} v+M_{t, s}^{\alpha, \beta} .
\end{aligned}
$$

5.3 Remark. Moreover, for $\alpha, \beta>\frac{1}{2}$, it follows from [8] and [22] that if we denote

$$
K_{\alpha}(t, v)=\alpha(2 \alpha-1) \int_{v}^{t} r^{2 \alpha-1}(r-v)^{\alpha-\frac{3}{2}} \mathrm{~d} r
$$

then it holds that

$$
X_{t, s}=\int_{0}^{t} \int_{0}^{s} K_{\alpha}(t, v) K_{\beta}(s, u) \mathrm{d} Z_{u, v}
$$


Denote

$$
R_{t, s}=\frac{\mathrm{d}}{\mathrm{d} \omega_{t}^{\alpha}} \frac{\mathrm{d}}{\mathrm{d} \omega_{s}^{\beta}} \int_{0}^{t} \int_{0}^{s} k_{\alpha}(t, v) k_{\beta}(s, u) b\left(X_{v, u}\right) \mathrm{d} u \mathrm{~d} v .
$$

Then we have the following:

- For every $(\alpha, \beta) \in(0,1)^{2}$ and if $b$ is Lipschitz, the sample paths of the process $R$ given by $(5.5)$ belong to $L^{2}\left([0,1]^{2}, \omega^{\alpha} \otimes \omega^{\beta}\right)$. This can be viewed in the same way as in Theorem 2.

- Clearly, the process $R$ is related to the process $Q$ (4.9) by

$$
R_{t, s}=\mathfrak{c}_{\alpha, \beta} t^{\alpha-\frac{1}{2}} s^{\beta-\frac{1}{2}} Q_{t, s}
$$

From (5.2) and (5.5) we obtain that

$$
Z_{t, s}=\theta \int_{0}^{t} \int_{0}^{s} R_{v, u} \mathrm{~d} \omega_{u}^{\beta} \mathrm{d} \omega_{v}^{\alpha}+M_{t, s}^{\alpha, \beta}
$$

and then the MLE for the parameter $\theta$ in (3.1) can be written as

$$
\theta_{t}=-\frac{\int_{0}^{t} \int_{0}^{t} R_{v, u} \mathrm{~d} M_{u, v}^{\alpha, \beta}}{\int_{0}^{t} \int_{0}^{t} R_{v, u}^{2} \mathrm{~d} \omega_{u}^{\beta} \mathrm{d} \omega_{v}^{\alpha}}
$$

In order to prove the strong consistency of the MLE, we derive an easier expression for the process $R$ (or, equivalently, for the process $Q$ ) appearing in the expression of the MLE in the linear case. In this case we have

$$
R_{t, s}=\frac{\mathrm{d}}{\mathrm{d} \omega_{t}^{\alpha}} \frac{\mathrm{d}}{\mathrm{d} \omega_{s}^{\beta}} \int_{0}^{t} \int_{0}^{s} k_{\alpha}(t, v) k_{\beta}(s, u) X_{v, u} \mathrm{~d} u \mathrm{~d} v
$$

We need first a more suitable expression of the process $R$ given by (5.9).

5.10 Proposition. For every $\alpha, \beta$ it holds that

$$
R_{t, s}=\frac{\lambda_{\alpha}^{*}}{2} \frac{\lambda_{\beta}^{*}}{2} \int_{0}^{t} \int_{0}^{s}\left(t^{2 \alpha-1}+v^{2 \alpha-1}\right)\left(s^{2 \beta-1}+u^{2 \beta-1}\right) \mathrm{d} Z_{u, v}
$$

with $\lambda_{\alpha}^{*}=\frac{\lambda^{\alpha}}{2(1-\alpha)}$.

Proof. We will restrict ourselves to the case $\alpha, \beta \leq \frac{1}{2}$. By (5.4) and (5.9) it holds that

$$
\begin{aligned}
\int_{0}^{t} \int_{0}^{s} R_{v, u} \mathrm{~d} \omega_{u}^{\beta} \mathrm{d} \omega_{v}^{\alpha} & =\int_{0}^{t} \int_{0}^{s} k_{\alpha}(t, v) k_{\beta}(s, u)\left(\int_{0}^{v} \int_{0}^{u} K_{\alpha}(v, b) K_{\beta}(u, a) \mathrm{d} Z_{a, b}\right) \mathrm{d} u \mathrm{~d} v \\
& =\int_{0}^{t} \int_{0}^{s} A_{\alpha}(b, t) A_{\beta}(b, s) \mathrm{d} Z_{a, b}
\end{aligned}
$$

where

$$
A_{\alpha}(b, t)=\int_{b}^{t} k_{\alpha}(t, v) K_{\alpha}(v, b) \mathrm{d} v
$$


Let us suppose a function $\Phi_{\alpha}(b, r)$ such that for every $b<t$,

$$
\int_{b}^{t} \Phi_{\alpha}(b, r) \mathrm{d} \omega_{r}^{\alpha}=A_{\alpha}(b, t) .
$$

Then it holds that

$$
\begin{aligned}
\int_{0}^{t} \int_{0}^{s} R_{v, u} \mathrm{~d} \omega_{u}^{\beta} \mathrm{d} \omega_{v}^{\alpha} & =\int_{0}^{t} \int_{0}^{s}\left(\int_{b}^{t} \Phi_{\alpha}(b, r) \mathrm{d} \omega_{r}^{\alpha}\right)\left(\int_{a}^{s} \Phi_{\beta}\left(a, r^{\prime}\right) \mathrm{d} \omega_{r^{\prime}}^{\beta}\right) \mathrm{d} Z_{a, b} \\
& =\int_{0}^{t} \int_{0}^{s}\left(\int_{0}^{r} \int_{0}^{r^{\prime}} \Phi_{\alpha}(b, r) \Phi_{\beta}\left(a, r^{\prime}\right) \mathrm{d} Z_{a, b}\right) \mathrm{d} \omega_{r^{\prime}}^{\beta} \mathrm{d} \omega_{r}^{\alpha}
\end{aligned}
$$

As a consequence

$$
R_{t, s}=\int_{0}^{t} \int_{0}^{s} \Phi_{\alpha}(t, v) \Phi_{\beta}(s, u) \mathrm{d} Z_{u, v} .
$$

On the other hand it has been proved in Lemma 3.1. of [9] that

$$
\Phi_{\alpha}(t, v)=\frac{\lambda^{*}}{2}\left(t^{2 \alpha-1}+v^{2 \alpha-1}\right) .
$$

The conclusion follows easily.

Let us finally discuss the asymptotic behavior of the estimator (5.8). We will actually prove the strong consistency of the MLEin the linear case $b(x)=x$ by trying to take advantage from the results contained in [9] (Proposition 2.2).

We first give the form of the solution in the case of linear drift.

5.12 Proposition. Let $X$ be the solution of the equation

$$
X_{t, s}=\theta \int_{0}^{t} \int_{0}^{s} X_{v, u} \mathrm{~d} u \mathrm{~d} v+W_{t, s}^{\alpha, \beta}, \quad t, s \in[0, T] .
$$

Then it holds that

$$
X_{t, s}=\int_{0}^{T} \int_{0}^{T} f\left(t_{0}, s_{0}, t, s\right) \mathrm{d} W_{t_{0}, s_{0}}^{\alpha, \beta}
$$

where

$$
f\left(t_{0}, s_{0}, t, s\right)=1_{[0, t]}\left(t_{0}\right) 1_{[0, s]}\left(s_{0}\right) \sum_{n \geq 0} \theta^{n} \frac{\left(t-t_{0}\right)^{n}\left(s-s_{0}\right)^{n}}{(n !)^{2}} .
$$

Proof. We use the kernel identification method as used for example recently in [15]. The solution $X_{t, s}$ is Gaussian and it admits the representation

$$
X_{t, s}=\int_{0}^{t} \int_{0}^{s} f\left(t_{0}, s_{0}, t, s\right) \mathrm{d} W_{t_{0}, s_{0}}^{\alpha, \beta}
$$


and thus the kernel $f$ satisfies

$$
f\left(t_{0}, s_{0}, t, s\right)=\theta \int_{0}^{t} \int_{0}^{s} f\left(t_{0}, s_{0}, v, u\right) \mathrm{d} v \mathrm{~d} u+1_{[0, t]}\left(t_{0}\right) 1_{[0, s]}\left(s_{0}\right) .
$$

for every $s, t, s_{0}, t_{0} \in[0, T]$. It is known (but one can check it easily) that the equation (5.16) admits the solution (5.15).

To prove the strong consistency, we will further assume that $W_{t, s}^{\alpha, \beta}=W_{t}^{\alpha} W_{s}^{\beta}$ where $W^{\alpha}$ and $W^{\beta}$ are two independent one-parameter fractional Brownian motion with Hurst index $\alpha, \beta \in(0,1)$ (clearly both processes have the same law).

5.17 Theorem. The MLE defined by (5.8) is strongly consistent, i.e for any real $\theta$

$$
\theta_{t} \rightarrow_{t \rightarrow \infty} \theta, \quad \text { a.s. }
$$

Proof. One can write

$$
\theta_{t}-\theta=\frac{\int_{0}^{t} \int_{0}^{t} R_{v, u} \mathrm{~d} M_{v, u}^{\alpha, \beta}}{\int_{0}^{t} \int_{0}^{t} R_{v, u} \mathrm{~d} \omega_{u}^{\beta} \mathrm{d} \omega_{v}^{\alpha}} .
$$

One the other hand one has by a version of the strong law of large numbers for twoparameters martingales that the quantity $\frac{\int_{0}^{t} \int_{0}^{s} R_{v, u} \mathrm{~d} M_{v, u}^{\alpha, \beta}}{\int_{0}^{t} \int_{0}^{s} R_{v, u} \mathrm{~d} \omega_{u}^{\beta} \mathrm{d} \omega_{v}^{\alpha}}$ converges to zero almost surely as $t, s \rightarrow \infty$ if

$$
\int_{0}^{t} \int_{0}^{s} R_{v, u} \mathrm{~d} \omega_{u}^{\beta} \mathrm{d} \omega_{v}^{\alpha}
$$

converges almost surely to infinity as $t, s \rightarrow \infty$. This fact will be showed in the rest of this proof.

By Proposition 5.12 we have

$$
X_{t, s}=\sum_{n \geq 0} \theta^{n} A_{n}(t) B_{n}(s)
$$

where

$$
A_{n}(t)=\int_{0}^{t} \frac{(t-v)^{n}}{n !} \mathrm{d} W_{v}^{\alpha} \quad \text { and } \quad B_{n}(s)=\int_{0}^{s} \frac{(s-u)^{n}}{n !} \mathrm{d} W_{u}^{\beta} .
$$

Therefore

$$
\begin{aligned}
Z_{t, s} & =\int_{0}^{t} \int_{0}^{s} k_{\alpha}(t, v) k_{\beta}(s, u) \mathrm{d} X_{v, u} \\
& =\sum_{n \geq 0} \theta^{n}\left(\int_{0}^{t} k_{\alpha}(t, v) \mathrm{d} A_{n}(v)\right)\left(\int_{0}^{s} k_{\beta}(s, u) \mathrm{d} B_{n}(u)\right) \\
& =: \sum_{n \geq 0} \theta^{n} C_{n}(t) D_{n}(s) .
\end{aligned}
$$


Now, Proposition 5.10 implies that

$$
\begin{aligned}
R_{t, s} & =\frac{\lambda_{\alpha}^{*}}{2} \frac{\lambda_{\beta}^{*}}{2} \int_{0}^{t} \int_{0}^{s}\left(t^{2 \alpha-1}+v^{2 \alpha-1}\right)\left(s^{2 \beta-1}+u^{2 \beta-1}\right) \mathrm{d} Z_{u, v} \\
& =: \sum_{n \geq 0} \theta^{n} E_{n}(t) F_{n}(s)
\end{aligned}
$$

where

$$
E_{n}(t)=\frac{\lambda_{\alpha}^{*}}{2} \int_{0}^{t}\left(t^{2 \alpha-1}+v^{2 \alpha-1}\right) \mathrm{d} C_{n}(v)
$$

and

$$
F_{n}(s)=\frac{\lambda_{\beta}^{*}}{2} \int_{0}^{s}\left(s^{2 \beta-1}+u^{2 \beta-1}\right) \mathrm{d} D_{n}(u) .
$$

We obtain that

$$
\begin{aligned}
& \int_{0}^{t} \int_{0}^{s} R_{v, u} \mathrm{~d} \omega_{u}^{\beta} \mathrm{d} \omega_{v}^{\alpha} \\
& =\int_{0}^{t} \int_{0}^{s}\left(\sum_{n \geq 0} \theta^{n} E_{n}(v) F_{n}(u)\right)^{2} \mathrm{~d} \omega_{u}^{\beta} \mathrm{d} \omega_{v}^{\alpha} \\
& =\sum_{n, m \geq 0} \theta^{n} \theta^{m}\left(\int_{0}^{t} E_{n}(v) E_{m}(v) \mathrm{d} \omega_{v}^{\alpha}\right)\left(\int_{0}^{s} F_{n}(u) F_{m}(u) \mathrm{d} \omega_{u}^{\beta}\right) .
\end{aligned}
$$

But the result of [9] (Proposition 2.2) implies that

$$
\sum_{n, m \geq 0} \theta^{n} \theta^{m} \int_{0}^{t} E_{n}(v) E_{m}(v) \mathrm{d} \omega_{v}^{\alpha} \rightarrow t \rightarrow \infty \infty \quad \text { a.s }
$$

and

$$
\sum_{n, m \geq 0} \theta^{n} \theta^{m} \int_{0}^{s} F_{n}(u) F_{m}(u) \mathrm{d} \omega_{u}^{\beta} \rightarrow_{s \rightarrow \infty} \infty \quad \text { a.s. }
$$

and this gives the convergence of (5.18) to infinity almost surely as $t, s \rightarrow \infty$.

\section{References}

[1] A. Ayache, S. Leger, M. Pontier (2002): Drap brownien fractionnaire. Potential Analysis, 17(1), pp. 31-34.

[2] A. J. Dorogovcep and P.S. Knopov (1979): An estimator of a two-dimensional signal from an observation with additive random noise. Theory of Probability and Mathematical Statistics, 17, pp. 67-86.

[3] M. Erraoui, D. Nualart and Y. Ouknine (2003): Hyperbolic stochastic partial differential equations with additive fractional Brownian sheet. Stochastics and Dynamics, 3, pp. 121-139. 
[4] X. Fernique (1974): Régularité des trajectoires de fonctions aléatoires gaussiennes. Lecture Notes in Mathematics (St. Flour), 480, pp. 2-95.

[5] A. Frieman (1975): Stochastic differential equations and applications. Academic Press.

[6] R. Jennane, W. J. Ohley, S. Majumdar and G. Lemineur (2001): Fractal analysis of bone x-ray tomographic microscopy projections. IEEE Trans. Med. Imag. , 20, pp. 443-449.

[7] C. Jung Lae and L. Chyi-Chying (1997): Sea clutter rejection in radar image using wavelets and fractals. Proc. INt. Conf. Image Processing., vol. 2, pp 354357.

[8] M. Kleptsyna, A. Le Breton and C. Roubaud (2000): Parameter estimation and optimal filtering for fractional type stochastic systems. Statistical Inference for stochastic processes, 3, pp. 173-182.

[9] M. Kleptsyna and A. Le Breton (2002): Statistical Analysis of the fractional Ornstein-Uhlenbeck process. Statistical Inference for stochastic processes, 5, pp. 229-248.

[10] A. Kukush, Y. Mishura and E. Valkeila (2005): Statistical inference with fractional Brownian motion. Statistical Inference for stochastic processes, 81, pp. 71-93.

[11] M. Ledoux and M. Talagrand (1990): Probability on Banach spaces. Springer.

[12] S. Leger (2001):Analyse stochastiques des signaux multifractaux et estimation de paramètres. Thèse de doctorat, U. de Orléans.

[13] H. Maitre and M. Pinciroli (1999): Fractal characterization of a hydrological basin using SAR satellite images. IEEE Trans. Geosc. Remote Sensing, 37, pp. 175-181.

[14] I. Norros, E. Valkeila, J. Virtamo (1999): An elementary approach to a Girsanov formula and other analytic results on fractional Brownian motion. Bernoulli, 5(4), pp. 571-587.

[15] I. Nourdin and C.A. Tudor (2006): On some fractionar linear equations. Stochastics, 78 (2), pp. 51-65.

[16] D. Nualart (1995): The Malliavin calculus and related topics. Springer.

[17] D. Nualart and Y. Ouknine (2002): Regularization of stochastic differential equation by fractional noise. Stoch. Proc. Applic., 102, pp. 103-116.

[18] B. Pesquet-Popescu and J. Lévy Véhel (2002): Stochastic fractal models for image processing models. IEEE Signal Proceesing Magazine, September 2002, pp. $48-62$. 
[19] B.L.S. Prakasa Rao (2003): Parameter estimation for linear stochastic differential equations driven by fractional Brownian motion. Random Operators Stoch. Eqs., 11(3), pp. 229-242.

[20] T. Sottinen (2003): Fractional Brownian motion in finance and queueing. Ph.D. Thesis, University of Helsinki. Introduction available electronically at ethesis.helsinki.fi/julkaisut/mat/matem/vk/sottinen/fraction.pdf

[21] T. Sottinen and C.A. Tudor (2005): On the equivalence of multiparameter Gaussian processes. Journal of Theoretical Probability, to appear.

[22] M. Tudor and C. Tudor (2002): On the multiparameter fractional Brownian motion. Preprint, 22 pp.

[23] C.A. Tudor and F. Viens (2003): Itô formula and local time for the fractional Brownian sheet. Electronic Journal of Probability, 8, paper 14, pp. 1-31.

[24] C.A. Tudor and F. Viens (2004): Ito formula for the fractional Brownian sheet using the extended divergence integral. Preprint, accepted for publication in Stochastics.

[25] C.A. Tudor and F. Viens (2005): Statistical Aspects of the fractional stochastic calculus. Preprint, accepted for publication in The Annals of Statistics.

[26] A.S. Üstunel (1985): An introduction to analysis on Wiener spave. Lecture Notes in Mathematics, 1610, Springer. 